\title{
ON P-INJECTIVE RINGS
}

\section{by GENNADI PUNINSKI, ROBERT WISBAUER and MOHAMED YOUSIF}

(Received 6 May, 1994)

1. Definitions and preliminary results. Throughout this paper $R$ will be an associative ring with unity and all $R$-modules are unitary. The right (resp. left) annihilator in $R$ of a subset $X$ of a module is denoted by $\mathbf{r}(X)$ (resp. $\mathbf{l}(X)$ ). The Jacobson radical of $R$ is denoted by $J(R)$, the singular ideals are denoted by $Z\left(R_{R}\right)$ and $Z\left({ }_{R} R\right)$ and the socles by $\operatorname{Soc}\left(R_{R}\right)$ and $\operatorname{Soc}\left({ }_{R} R\right)$. For a module $M, E(M)$ and $\operatorname{PE}(M)$ denote the injective and pure-injective envelopes of $M$, respectively. For a submodule $A \subseteq M$, the notation $A \subseteq \subseteq^{\oplus} M$ will mean that $A$ is a direct summand of $M$.

A module $M_{R}$ is called $p$-injective if for every $a \in R$, every $R$-linear map from $a R$ to $M$ can be extended to an $R$-linear map from $R$ to $M$. $R$ is called right p-injective if $R_{R}$ is p-injective. Recall that a module $M_{R}$ is called uniserial if its submodules are linearly ordered by inclusion and serial if it is a direct sum of uniserial submodules. A ring $R$ is right uniserial (serial) if $R_{R}$ is uniserial (serial).

We record some well-known results on serial and p-injective rings.

LemMa $1.1[5,6]$. Let $R$ be any ring.

(1) $R$ is right $p$-injective if and only if $1(\mathbf{r}(a))=R a$ for every $a \in R$.

(2) If $R$ is right $p$-injective then $J(R)=Z\left(R_{R}\right)$.

(3) If $R$ is left uniserial then $R$ is right p-injective if and only if $J(R)=Z\left(R_{R}\right)$.

(4) If $R$ is right $p$-injective and $A, B_{1}, \ldots, B_{n}$ are two-sided ideals of $R$ then

$$
A \cap\left(B_{1} \oplus \ldots \oplus B_{n}\right)=\left(A \cap B_{1}\right) \oplus \ldots \oplus\left(A \cap B_{n}\right) .
$$

Lemma 1.2 [11, p. 200, Theorem 3.3]. Let $R$ be a serial ring, $P$ a finitely generated projective $R$-module, and $M$ a finitely generated submodule of $P$. Then there is a decomposition $P=P_{1} \oplus \ldots \oplus P_{n}$ with indecomposables $P_{i}$ such that

$$
M=\left(M \cap P_{1}\right) \oplus \ldots \oplus\left(M \cap P_{n}\right) .
$$

The next two statements are proved using model theory for modules.

LEMMA 1.3 [3]. Let $R$ be an arbitrary ring and $M$ a finitely presented module over $R$. Then $\operatorname{PE}(M)$ is indecomposable if and only if $M$ has a local endomorphism ring.

LEMMA 1.4 [7]. Let $R$ be a serial ring and $M$ a pure-injective indecomposable module over $R$. Then either $M$ is injective or, for every primitive idempotent $e \in R$ and every nonzero element $m \in M e$, there exists an element $r \in R$ such that $m \in E(M) r e$ and $m \notin$ Mre.

Lemma 1.5 [5, Corollary 2.2, Theorem 2.3]. Let $R$ be a semiperfect right p-injective ring with $\operatorname{Soc}\left(R_{R}\right)$ essential as a right ideal in $R$. Then $\operatorname{Soc}\left(R_{R}\right)=\operatorname{Soc}\left({ }_{R} R\right)$ is essential as a left ideal and $Z\left(R_{R}\right)=J(R)=Z\left({ }_{R} R\right)$.

Recall that a right $R$-module $M$ is called fp-injective if every $R$-linear map from a finitely generated submodule of a free $R$-module $F$ to $M$ can be extended to an $R$-linear map from $F$ to $M$. Evidently every fp-injective module is p-injective and the converse is 
true for some classes of rings including serial rings, see [8]. In the serial ring case we give a short proof of this fact using the above cited Warfield's result.

Lemma 1.6. Every right p-injective module $M$ over a serial ring $R$ is fp-injective.

Proof. Let $N$ be a finitely generated submodule of a free module $P$ of finite rank and $f$ a homomorphism from $N$ into $M$. In view of Lemma 1.2 we may assume that $N$ is a finitely generated submodule of an indecomposable projective module $e R$ for some primitive idempotent $e \in R$. Since $e R$ is uniserial, it follows that $N$ is cyclic. Now the existence of the desired extension follows from p-injectivity of $M$.

2. Serial p-injective rings. Now we formulate our criteria for serial rings to be right p-injective.

THEOREM 2.1. For a serial ring $R$ with a complete set of primitive orthogonal idempotents $\left\{e_{1}, \ldots, e_{n}\right\}$ the following conditions are equivalent:

(a) $R$ is right p-injective;

(b) $R$ is right fp-injective;

(c) $J(R)=Z\left(R_{R}\right)$

(d) for any pair of indices $i, j \leq n$ and any $r \in R$ with $0 \neq e_{i} r e_{j} \in J\left(R e_{j}\right)$ there exist $s \in R$ and $k \leq n$, such that $e_{j} s e_{k} \neq 0$ and $e_{i} r e_{j} s e_{k}=0$.

Proof. The equivalence between (a) and (b) follows from Lemma 1.6 and the implication (b) $\Rightarrow$ (c) follows from Lemma 1.1.

(c) $\Rightarrow$ (d). If $0 \neq e_{i} r e_{j} \in J\left(R e_{j}\right)$ then $e_{i} r e_{j} \in J(R)=Z\left(R_{R}\right)$, hence $\mathbf{r}\left(e_{i} r e_{j}\right)$ is essential in $R_{R}$ and $\mathbf{r}\left(e_{i} r e_{j}\right) \cap e_{j} R \neq 0$. It follows that $e_{i} r e_{j} s=0$ for some nonzero $e_{j} s \in e_{j} R$. Since $e_{j} R$ is uniserial and $e_{j} s R=e_{j} s e_{1} R+\ldots+e_{j} s e_{n} R$ we obtain $e_{j} s R=e_{j} s e_{k} R$ for some $k$ and $e_{j} s e_{k}$ is the desired element.

(d) $\Rightarrow$ (a). Suppose that $R_{R}$ is not p-injective. Then $e_{j} R$ is not p-injective as a right $R$-module for some $j$. Let $M$ be the pure-injective envelope of $e_{j} R$. Since $e_{j} R$ has a local (in fact uniserial) endomorphism ring it follows from Lemma 1.3 that $M$ is an indecomposable pure-injective module. Now if $M$ is injective, it will follow that $e_{j} R$ is $f p$-injective since it is a pure submodule of $M$, a contradiction. By Lemma 1.4, applied to the element $e_{j} \in M e_{j}$, we can find an element $r \in R$ such that $e_{j} \in E(M) r e_{j}$ and $e_{j} \notin M r e_{j}$. If $r e_{j} \notin J\left(R e_{j}\right)$ then $t r e_{j}=e_{j}$ for some $t \in R$. Now, $e_{j} t \in e_{j} R \subseteq M$ implies $e_{j}=e_{j} t . r e_{j} \in M r e_{j}$, a contradiction. Hence we may assume $r e_{j} \in J\left(R e_{j}\right)$. Since $R e_{i} r e_{j}=R r e_{j}$, for some $i$, it follows $e_{i} r e_{j} \in J\left(R e_{j}\right)$ and hence by assumption $e_{i} r e_{j} s e_{k}=0$ for some $k$ and some $s \in R$.

Since $e_{j} \in E(M) r e_{j}$ we obtain $e_{j}=m r e_{j}$ for some $m \in E(M)$. Multiplying this equality by $e_{j} s e_{k}$ from the right side we obtain $e_{j} s e_{k}=m r e_{j} \cdot e_{j} s e_{k}=0$, a contradiction.

COROLlaRY 2.2. Let $R$ be a serial right p-injective ring with essential right socle. Then $R$ is left p-injective with essential left socle.

Proof. From Lemma 1.5 we obtain $Z\left(R_{R}\right)=J(R)=Z\left({ }_{R} R\right)$ and the socle of $R$ is essential in ${ }_{R} R$. From Theorem 2.1 it follows that $R$ is left $\mathrm{p}$-injective.

EXAMPLE 2.3. Let $F$ be an arbitrary field and consider the ring

$$
R=\left[\begin{array}{ll}
F & F \\
0 & F
\end{array}\right]
$$

Then $R$ is a (two-sided) serial artinian ring which is neither left nor right $p$-injective. 
Proof. We check this for the right side only. We have $e_{12} \in J\left(R e_{2}\right) \cap e_{1} R e_{2}$ and $e_{12} s \neq 0$ for every nonzero element $s \in e_{2} R$ which contradicts (d) of Theorem 2.1.

Next we provide an example of a ring $R$ which is a right uniserial right artinian right duo left $p$-injective ring which is neither right p-injective nor left uniform. Also every non-invertible element of $R$ has an essential left and right annihilator. Recall that a ring $R$ is right duo if every right ideal of $R$ is two-sided.

EXAMPLE 2.4. Let $K$ be a field and $K(x)$ the field of rational functions over $K$. Let $\alpha$ be an endomorphism of $K(x)$ which sends $x$ to $x^{2}$. Clearly the image of $\alpha$ is $K\left(x^{2}\right)$. Let $R$ be a matrix ring of the form

$$
\left\{\left[\begin{array}{cc}
\alpha(a) & b \\
0 & a
\end{array}\right]: a, b \in K(x)\right\}
$$

Clearly

$$
\left[\begin{array}{cc}
0 & K(x) \\
0 & 0
\end{array}\right]
$$

is the unique non-trivial right ideal of $R$. If we view $K(x)$ as a vector space over $K\left(x^{2}\right)$ then every proper left ideal of $R$ has the form

$$
\left[\begin{array}{ll}
0 & V \\
0 & 0
\end{array}\right]
$$

where $V$ is a subspace of $K(x)$. It is easy to check that for every $a \in J$, the Jacobson radical of $R, \mathbf{r}(a)=\mathbf{l}(a)=J$. Clearly $R$ is right artinian right uniserial right duo and not left uniserial. It follows from Lemma 1.1 that $R$ is left p-injective and not right p-injective.

3. Semiperfect p-injective rings. In this section we show that semiperfect right p-injective right duo rings are right continuous. Recall that a module $M_{R}$ is called continuous if it satisfies the following two conditions: (C1) every submodule of $M$ is essential in a direct summand, and (C2) if $A$ and $B$ are submodules of $M$ with $A \cong B$ and $B \subseteq^{\oplus} M$ then $A \subseteq^{\oplus} M$.

In [5, Theorem 1.2], it was shown that if $R_{R}$ is right p-injective then $R_{R}$ satisfies the C2-condition. In particular, if $A$ and $B$ are right ideals of $R$ with $A \subseteq{ }^{\oplus} R_{R}, B \subseteq^{\oplus} R_{R}$ and $A \cap B=0$ then $A \oplus B \subseteq^{\oplus} R_{R}$. If $R$ is right duo we have the following more general result which is of independent interest.

THEOREM 3.1. Let $R$ be a right p-injective right duo ring. If $A$ and $B$ are right ideals of $R$ with $A \subseteq{ }^{\oplus} R_{R}$ and $B \subseteq^{\oplus} R_{R}$ then $(A \cap B) \subseteq{ }^{\oplus} R_{R}$ and $(A+B) \subseteq{ }^{\oplus} R_{R}$.

Proof. Write $R=A \oplus A_{1}=B \oplus B_{1}$ for some right ideals $A_{1}$ and $B_{1}$ of $R$. By Lemma 1.1, $B=B \cap\left(A \oplus A_{1}\right)=(B \cap A) \oplus\left(B \cap A_{1}\right)$. Hence

$$
R=(B \cap A) \oplus\left(B \cap A_{1}\right) \oplus B_{1}
$$

and so $(A \cap B) \subseteq^{\oplus} R_{R}$. Also

$$
A+B=A+\left((B \cap A) \oplus\left(B \cap A_{1}\right)\right)=(A+(B \cap A)) \oplus\left(B \cap A_{1}\right)=A \oplus\left(B \cap A_{1}\right) .
$$


Since both $A$ and ( $B \cap A_{1}$ ) are summands of $R_{R}$, it follows from the remark preceding the theorem that $A \oplus\left(B \cap A_{1}\right)$ is a summand of $R_{R}$ and so $A+B$ is also a summand of $R_{R}$.

Lemma 3.2. Let $R$ be a local right p-injective ring. Then for any non-zero (two-sided) ideals $I$ and $J$ of $R, I \cap J \neq 0$.

Proof. Suppose that $I \cap J=0$ and let $0 \neq u \in I, 0 \neq v \in J$. Define the map

$$
\varphi:(u+v) R \rightarrow R, \quad(u+v) r \mapsto u r .
$$

Clearly $\varphi$ is a well defined $R$-homomorphism. By right p-injectivity, $\varphi$ is given by left multiplication by an element $t \in R$. Hence $t(u+v)=u$, and so $(1-t) u=t v=0$. Since $R$ is a local ring it follows that $u=0$ or $v=0$, a contradiction.

Corollary 3.3. Suppose $R$ is a local right p-injective right duo ring. Then $R$ is right uniform.

REMark 3.4. Note that without the condition "right duo" the above result is not true. The ring $R$ given in Example 2.4 is a local left p-injective ring which is not left uniform.

THEOREM 3.5. Suppose $R$ is a semiperfect right duo right p-injective ring. Then $R$ is right continuous.

Proof. By Corollary 3.3, clearly $R$ is a direct sum of local right uniform rings $R_{i}$. By [5, Theorem 1.2], any right p-injective ring satisfies the C2-condition. We only need to show that $R_{R}$ satisfies the C1-condition. Let $A$ be a non-zero right ideal of $R$ and write $R=R_{1} \oplus \ldots \oplus R_{n}$. By Lemma 1.1, without loss of generality we may write $A=\left(A \cap R_{1}\right) \oplus \ldots \oplus\left(A \cap R_{k}\right)$, for some $k \leq n$ with $A \cap R_{i} \neq 0, \quad 1 \leq i \leq k$. Since each $A \cap R_{i}$ is essential as a right ideal in $R_{i}, 1 \leq i \leq k$, it follows that $A_{R}$ is essential in $R_{1} \oplus \ldots \oplus R_{k} \subseteq^{\oplus} R_{R}$.

Remark 3.6. Note that the ring $R$ given in Example 2.4 is a left p-injective right artinian ring which is not left finite dimensional. Hence $R$ can not be left continuous.

4. Completely p-injective rings. A ring $R$ is called completely right p-injective (right $c p$-injective) if every ring homomorphic image of $R$ is right p-injective. $R$ is called $c p$-injective if it is both left and right $\mathrm{cp}$-injective. In this section, for right duo rings, we give a characterization for serial rings with nil Jacobson radical in terms of cp-injectivity. Recall that a module $M$ is said to be distributive if its lattice of submodules is distributive: for all $A, B, C \subset M, A \cap(B+C)=A \cap B+A \cap C$.

THEOREM 4.1. Let $R$ be a right cp-injective ring. Then the lattice of two-sided ideals of $R$ is distributive.

Proof. Suppose the lattice of two-sided ideals of $R$ is a non-distributive (modular) lattice. It follows from [2, Theorem 2] that it contains a minimal non-distributive modular sublattice consisting of five elements. Hence we can find three noncomparable two-sided ideals $I, J$ and $K$ in $R$ such that $I \cap J=I \cap K=J \cap K$ and $I+J=I+K=J+K$. Then factorizing by the common intersection we may suppose that all these sums are direct and all these intersections are zero. Now by Lemma 1.1 it follows that $0 \neq I=I \cap(J \oplus K)=$ $(I \cap J) \oplus(I \cap K)=0$, a contradiction.

COROLlaRY 4.2. Every right duo right cp-injective ring is right and left distributive. 
Proof. The right distributivity follows from the above theorem and we can apply the following result from [9, Corollary 2.10$]$ : every right distributive right $\mathrm{p}$-injective ring is left distributive.

Recall that a ring $R$ is strongly regular if for every $a \in R$ there exists $b \in R$ such that $a=b a^{2}$.

LEMMA 4.3. For a ring $R$ the following are equivalent:

(a) $R$ is strongly regular;

(b) $R$ is right p-injective with no non-zero nilpotent elements;

(c) $R$ is a semiprime right p-injective right duo ring.

Proof. (a) $\Rightarrow$ (b), (c) is standard.

(c) $\Rightarrow$ (a). We adopt the argument given in Example 6 of [5]. Let $a \in R$ and set $T=a R \cap \mathbf{r}(a)$. Then clearly $T$ is a two-sided ideal of $R$ with $T^{2}=0$. Since $R$ is semiprime, $T=0$ and hence $\mathbf{r}\left(a^{2}\right)=\mathbf{r}(a)$. By Lemma 1.1 we get $R a=R a^{2}$ and hence $R$ is (strongly) regular.

(b) $\Rightarrow$ (a). Note that in rings without non-zero nilpotent elements for every $a \in R$, $\mathbf{r}(a)=\mathbf{l}(a)$. Now the same argument as before applies

REMARK 4.4. More results of the type given in Lemma 4.3 may be found in some of Yue Chi Ming's work on p-injectivity (e.g. [14]).

A ring $R$ is $\pi$-regular if every descending chain of the form $a R \supseteq a^{2} R \supseteq \ldots$ becomes stationary.

Lemma 4.5. Let $R$ be right duo and right cp-injective. Then $R$ is $\pi$-regular.

Proof. Let $a \in R$ and consider the following ascending chain of right annihilators $\mathbf{r}(a) \subseteq \mathbf{r}\left(a^{2}\right) \subseteq \ldots$ Let $I=\bigcup_{i=1}^{\infty} \mathbf{r}\left(a^{i}\right)$ and consider the ring $\bar{R}=R / I$. Clearly $\mathbf{r}_{\bar{R}}(\bar{a})=\overline{0}$ and hence it follows from Lemma 1.1 that $\bar{R} \bar{a}=\bar{R}$. So $1-s a \in \mathbf{r}\left(a^{m}\right)$ for some $s \in R$ and $m>0$. Since $R$ is right duo there exists $t \in R$ such that $s a=a t$ and hence $a^{m}=a^{m+1} t$ from which we infer that $R$ is $\pi$-regular.

THEOREM 4.6. For a right duo ring $R$ the following conditions are equivalent:

(a) $R$ is right cp-injective with no infinite set of orthogonal idempotents;

(b) $R$ is cp-injective with no infinite set of orthogonal idempotents;

(c) $R$ is a finite direct sum of (two-sided) uniserial rings with nil Jacobson radical.

Proof. (a) $\Rightarrow$ (c). By Lemma 4.5, $R$ is $\pi$-regular and hence $J(R)$ is a nil ideal and so idempotents can be lifted modulo $J(R)$. By assumption and Lemma 4.3, it follows that $R / J(R)$ is semisimple artinian and hence $R$ is semiperfect. Hence $R=R_{1} \oplus \ldots \oplus R_{n}$ where each $R_{i}$ is a local ring which is left and right distributive by Corollary 4.2. Since local right distributive rings are right uniserial we are done.

(c) $\Rightarrow$ (b). We may assume that $R$ is uniserial with nil radical $J$. Let $I$ be any (two-sided) ideal of $R$ and consider the ring $\bar{R}=R / I$. Clearly, every element of $J(\bar{R})$ has a nonzero left and right annihilator. Hence by [6, Lemma 1], $\bar{R}$ is right and left p-injective.

(b) $\Rightarrow(a)$ is trivial. 
Notice that any von Neumann regular ring which is not right noetherian is cp-injective with an infinite set of orthogonal idempotents.

ACKNOWLEDGEMENT. This paper was written while the first and third authors were visiting the Mathematical Institute of the University of Düsseldorf. Both authors would like to thank the Institute for the warm hospitality. The first author was supported by Heinrich-Hertz-Stiftung des Ministerium für Wissenschaft und Forschung des Landes Nordrhein-Westfalen. The third author was supported by a research grant from the Ohio State University.

\section{REFERENCES}

1. V. Camillo, Commutative rings whose principal ideals are annihilators, Portugal. Math. $\mathbf{4 6}$ (1989), 33-37.

2. G. Grätzer, Lattice theory, (Freeman \& Company, 1971).

3. I. Herzog, A test for finite representation type, J. Pure Appl. Alg. 95 (1994), 151-182. 94-109.

4. B. J. Müller and S. Singh, Uniform modules over serial rings, J. Algebra 144 (1991),

5. W. K. Nicholson and M. F. Yousif, Principally injective rings, J. Algebra 174 (1995), 77-93.

6. W. K. Nicholson and M. F. Yousif, On completely principally injective rings, Bull. Austral. Math. Soc. 49 (1994), 513-518.

7. G. Puninski, Pure injective modules over right noetherian serial rings, Comm. Algebra 23 (1995), 1579-1592. (1994).

8. G. Puninski, M. Prest and Ph. Rothmaler, Rings described by various purities, preprint

9. G. Puninski and R. Wisbauer, $\Sigma$-pure injective modules over left duo and left distributive rings, preprint (1994). 203-212.

10. E. A. Rutter, Rings with the principal extension property, Comm. Algebra 3 (1975), $187-222$.

11. R. B. Warfield, Jr., Serial rings and finitely presented modules, J. Algebra 37 (1975),

12. R. Wisbauer, Foundations of module and ring theory (Gordon and Breach, 1991). 350-357.

13. M. H. Wright, Right locally distributive rings in Ring theory, (World Scientific, 1993),

14. R. Yue Chi Ming, On injectivity and p-injectivity, J. Math. Kyoto Univ. 27 (1987), 439-452.

Gennadi Puninski

RusSian SOCIAL Institute

LOSIONOSTROVSKAJA, 24

107150 Moscow, Russia

Mohamed Yousif

Department of Mathematics

Ohio State University

LiMA, Оніо 45804, USA
Robert Wisbauer

Mathematisches INSTITUT der UNIVERSitäT

UNIVERSITÄTSSTR. 1

40225 DÜSSELDORF, GERMANY 